\title{
Pacific
}

Journal of

Mathematics

\section{ON AN OVERDETERMINED ELLIPTIC PROBLEM}

Laurent Hauswirth, Frédéric Hélein and Frank PaCARD 


\title{
ON AN OVERDETERMINED ELLIPTIC PROBLEM
}

\author{
Laurent Hauswirth, Frédéric Hélein And Frank PACARd
}

\begin{abstract}
A smooth flat Riemannian manifold is called an exceptional domain if it admits positive harmonic functions having vanishing Dirichlet boundary data and constant (nonzero) Neumann boundary data. In analogy with minimal surfaces, a representation formula is derived and applied to the classification of exceptional domains. Some interesting open problems are proposed along the way.
\end{abstract}

\section{Introduction}

Given an $m$-dimensional Riemannian manifold $(M, g)$ and a smooth bounded domain $\Omega$ in $M$, we denote by $\lambda_{1}(\Omega)$ the first eigenvalue of the Laplace-Beltrami operator under an identically zero Dirichlet boundary condition. The critical points of the functional

$$
\Omega \mapsto \lambda_{1}(\Omega)
$$

under the volume constraint $\operatorname{Vol} \Omega=\alpha$, where $\alpha \in(0, \operatorname{Vol} M)$ is fixed, are called extremal domains. Smooth extremal domains are characterized by the property that the eigenfunctions associated with the first eigenvalue of the Laplace-Beltrami operator have constant Neumann boundary data [Soufi 2007]. In other words, a smooth domain is extremal if and only if there exists a positive function $u_{1}$ and a constant $\lambda_{1}$ such that

$$
\Delta_{g} u_{1}+\lambda_{1} u_{1}=0
$$

in $\Omega$ with $u_{1}=0$ and $\nabla_{n} u_{1}$ constant on $\partial \Omega$, where $n$ denotes the inward unit normal vector to $\partial \Omega$.

The theory of extremal domains is very reminiscent of the theory of constant mean curvature surfaces or hypersurfaces. To give some credit to this assertion, we recall that J. Serrin [1971] proved that the only compact, smooth, extremal domains in Euclidean space are round balls, paralleling the well-known result of Alexandrov asserting that round spheres are the only (embedded) compact constant mean curvature hypersurfaces in Euclidean space. More recently, F. Pacard and

We thank Romain Dujardin and Charles Favre for useful discussions. F. Pacard is partially supported by the ANR-08-BLANC-0335-01 grant.

MSC2000: primary 35N25, 35J25, 35R35; secondary 53A10.

Keywords: harmonic function, overdetermined elliptic problem, extremal domain, minimal surface. 
P. Sicbaldi [2009] proved the existence of extremal domains close to small geodesic balls centered at critical points of the scalar curvature function, paralleling an earlier result of R. Ye [1991], which provides constant mean curvature topological spheres (with high mean curvature) close to small geodesic spheres centered at nondegenerate critical points of the scalar curvature function.

We propose the following:

Definition 1.1. A smooth domain $\Omega \subset \mathbb{R}^{m}$ is said to be an exceptional domain if it supports positive harmonic functions having identically zero Dirichlet boundary data and constant (nonzero) Neumann boundary data. Any such harmonic function is called a roof function.

Exceptional domains arise as limits under scaling of sequences of extremal domains, just like minimal surfaces arise as limits under scaling of sequences of constant mean curvature surfaces. As explained above, there is a formal correspondence between extremal domains and constant mean curvature surfaces. In this note, we try to explain that there is also a strong analogy between exceptional domains and minimal surfaces. More generally, we propose:

Definition 1.2. An $m$-dimensional flat Riemannian manifold $M$ is said to be exceptional if it supports positive harmonic functions having identically zero Dirichlet boundary data and constant (nonzero) Neumann boundary data. Any such harmonic function is called a roof function.

Our results raise the problem of the classification of (unbounded) smooth $\mathrm{m}$ dimensional exceptional manifolds. In trying to address this classification problem, we provide a Weierstrass-type representation formula for exceptional flat surfaces. When the dimension $m=2$, we give nontrivial examples of exceptional domains that are embedded in $\mathbb{R}^{2}$, and we prove a half-space result for exceptional domains that are conformal to a half-plane.

\section{A nontrivial example of an exceptional domain in $\mathbb{R}^{2}$}

The property of being an exceptional domain is preserved under the action of the group of similarities of $\mathbb{R}^{m}$ (generated by isometries and dilations). We first give trivial examples of exceptional domains in $\mathbb{R}^{m}$ :

(i) The half-space $\left\{x=\left(x_{1}, \ldots, x_{m}\right) \in \mathbb{R}^{m}: x_{1}>0\right\}$ is an exceptional domain in $\mathbb{R}^{m}$, since the function $u(x)=x_{1}$ is a positive harmonic function with identically zero Dirichlet boundary data and constant Neumann boundary data.

(ii) The complement of a ball of radius 1 in $\mathbb{R}^{m}$ is an exceptional domain since the function $u$ defined by $u(x):=\log |x|$ when $m=2$ and by $u(x):=1-|x|^{2-m}$ when $m \geq 3$ is positive, harmonic, and has 0 Dirichlet and constant Neumann data on the unit sphere. 
(iii) The product $\Omega \times \mathbb{R}^{k}$ is an exceptional domain in $\mathbb{R}^{m}$ provided $\Omega \subset \mathbb{R}^{m-k}$ is an exceptional domain in $\mathbb{R}^{m-k}$.

In dimension $m=2$, there exists (up to a similarity) at least one other exceptional domain. To describe this domain, we make use of the invariance of the Laplace operator under conformal transformations. The idea is that there exists a (somehow natural) unbounded, positive harmonic function $U$ with identically zero Dirichlet boundary condition on an infinite strip in $\mathbb{R}^{2}$. This function does not have constant Neumann data, but we can then look for a conformal transformation $h$ which has the property that the pullback of the harmonic function $U$ by $h$ has constant Neumann boundary data on the boundary of the image of the strip by $h$.

To proceed, it is convenient to identify $\mathbb{R}^{2}$ with the complex plane $\mathbb{C}$.

Proposition 2.1. The domain $\Omega:=\{w \in \mathbb{C}:|\operatorname{Im} w|<\pi / 2+\cosh (\operatorname{Re} w)\}$ is an exceptional domain.

To prove this result, we define the infinite strip

$$
S:=\{z \in \mathbb{C}: \operatorname{Im} z \in(-\pi / 2, \pi / 2)\}
$$

and the mapping

$$
F(z):=z+\sinh z .
$$

Observe that $\Omega=F(S)$. The proof of Proposition 2.1 follows from the next two lemmas.

Lemma 2.2. The mapping $F$ is a conformal diffeomorphism from $S$ into $\Omega$.

Proof. We can write

$$
F(z)-F\left(z^{\prime}\right)=\left(z-z^{\prime}\right) \int_{0}^{1}\left(1+\cosh \left(t z+(1-t) z^{\prime}\right)\right) d t .
$$

In particular

$$
\left\langle z-z^{\prime}, F(z)-F\left(z^{\prime}\right)\right\rangle=\left|z-z^{\prime}\right|^{2}\left(1+\int_{0}^{1} \operatorname{Re} \cosh \left(t z+(1-t) z^{\prime}\right) d t\right)
$$

where $\langle\cdot, \cdot\rangle$ denotes the scalar product in $\mathbb{C}$. Now, for all $x+i y \in S$, we have

$$
\operatorname{Re} \cosh (x+i y)=\cosh x \cos y \geq 0 .
$$

This, together with (2-1), implies immediately that $F$ restricted to $S$ is injective. Also,

$$
\left|\partial_{z} \Lambda(z)\right|^{2}=|1+\cosh z|^{2}=(\cosh x+\cos y)^{2} .
$$

Therefore $\partial_{z} F$ does not vanish in $S$. Thus $F$ is a local diffeomorphism, and because the mapping $F$ is holomorphic, it is conformal. 
We define the real-valued function $u$ on $\Omega$ by the identity

$$
u(F(z))=\operatorname{Re} \cosh z \quad \text { for all } z \in S .
$$

Lemma 2.3. The function $u$ is harmonic and positive in $\Omega$, vanishes and has constant Neumann boundary data on $\partial \Omega$.

Proof. The function $W$ defined in $\mathbb{C}$ by $W(z):=\operatorname{Re} \cosh z$ is harmonic. Indeed, as mentioned in the proof of the previous lemma, $W(x+i y)=\cosh x \cos y$. Hence $W$ is both harmonic and positive in $S$, and vanishes on $\partial S$. The mapping $F$ being a conformal diffeomorphism from $S$ to $\Omega$, we conclude the function $u$ is both harmonic and positive in $\Omega$, and vanishes on $\partial \Omega$. We claim that $u$ has constant Neumann data on $\partial \Omega$. Indeed, by definition,

$$
u(F(z))=\frac{1}{2}(\cosh z+\cosh \bar{z}) .
$$

Since $F$ is holomorphic, differentiation with respect to $z$ yields

$$
2 \partial_{z} u(F(z))=\frac{\sinh z}{1+\cosh z} .
$$

Therefore

$$
|\nabla u|^{2}(F(z))=\frac{\cosh x-\cos y}{\cosh x+\cos y},
$$

where $z=x+i y$. On $\partial \Omega$, we have $y= \pm \pi / 2$ and hence $|\nabla u| \equiv 1$. Since we already know that $u=0$ on $\partial \Omega$, we conclude that $u$ has constant Neumann boundary data.

Lemmas 2.2 and 2.3 complete the proof that $\Omega=F(S)$ is an exceptional domain in $\mathbb{R}^{2}$ with roof function $u$.

Remark 2.4. We suspect that this example generalizes to any dimension $m \geq 3$ : specifically, there should exist a rotationally symmetric exceptional domain in $\mathbb{R}^{m}$ for all $m \geq 3$.

\section{Toward a global representation formula}

Let $M$ be an exceptional flat surface (an exceptional domain of dimension 2) with smooth boundary $\partial M$. Let $\tilde{M}$ be its universal cover and let $\partial \tilde{M}$ be the preimage of $\partial M$ by the covering map $\tilde{M} \rightarrow M$. In the following, we exclude the uninteresting case where $\partial M=\varnothing$.

By assumption, $M$ is a flat surface. Hence $\tilde{M}$ is naturally endowed with a flat Riemannian metric $g$ and hence with an induced complex structure, which is conformal to the standard one. Also, there exists an orientation-preserving isometric immersion $F:(\tilde{M}, g) \rightarrow\left(\mathbb{C}, g_{\mathbb{C}}\right)$, where $g_{\mathbb{C}}$ is the canonical Euclidean metric on 
$\mathbb{C}$; this induces a smooth immersion of $\partial \tilde{M}$, Observe that $F$ is holomorphic and that $\|d F\|_{g}=1$ in $\tilde{M} \cup \partial \tilde{M}$. We define the holomorphic (1,0)-form

$$
\Phi:=d F=\partial_{z} F d z .
$$

Observe that $\Phi$ does not vanish and admits a smooth extension to $\tilde{M} \cup \partial \tilde{M}$.

We let $u: M \rightarrow \mathbb{R}^{+}$be a roof function on $M$ and, with a slight abuse of notation, we denote its lift also by $u: \tilde{M} \rightarrow \mathbb{R}^{+}$. The roof function $u$ can be normalized so that

$$
\|\nabla u\|_{g}=1
$$

on $\partial M$. We consider the harmonic conjugate function $v: \tilde{M} \rightarrow \mathbb{R}$ (uniquely defined up to some additive constant) that is the solution of

$$
\partial_{z}(u-i v)=0 \quad\left(\text { and hence } \partial_{\bar{z}}(u+i v)=0\right) .
$$

We set

$$
U:=u+i v .
$$

Recall that $U$ is a holomorphic function from $\tilde{M}$ into $\mathbb{C}$. The property that $u$ takes positive values in $M$ and vanishes on $\partial M$ can be translated into the fact that $U$ maps $\tilde{M}$ to $\mathbb{C}^{+}:=\{w \in \mathbb{C}: \operatorname{Re} w>0\}$ and $\partial \tilde{M}$ to $i \mathbb{R}$. Since $\Phi \neq 0$ on $\tilde{M}$, there exists a unique holomorphic function $h$ on $\tilde{M}$ such that $d U=\partial_{z} U d z=h \Phi$. We deduce from the fact that $u$ vanishes on $\partial \tilde{M}$ and from (3-1) that $\nabla_{n} U=1$, where $n$ denotes the inward unit normal vector to $\partial \tilde{M}$. Hence

$$
\left\|\partial_{z} U\right\|_{g}=1 \quad \text { on } \partial \tilde{M} \text {. }
$$

Now, condition (3-1) translates into the fact that $\|\Phi\|_{g}=\|d F\|_{g}=1=\|d U\|_{g}$ on $\partial \tilde{M}$. Clearly, this is equivalent to the fact that $|h|=1$ on $\partial \tilde{M}$. Therefore, we end up with the following data:

(i) An oriented, simply connected complex surface $\tilde{M}$ with smooth boundary $\partial \tilde{M}$.

(ii) A holomorphic function $U$, defined on $\tilde{M}$, which takes values in $\mathbb{C}^{+}$and maps д $\tilde{M}$ into $i \mathbb{R}$.

(iii) A holomorphic function $h$, defined on $\tilde{M}$, such that $|h|=1$ on $\partial \tilde{M}$, and for which the 1 -form $\Phi$ defined by $\Phi:=(1 / h) d U$ does not vanish on $\tilde{M}$.

By analogy with the theory of minimal surfaces, we call these data the Weierstrasstype representation formula for exceptional flat surfaces.

Conversely, given a set of such data, we can define the map $F: \tilde{M} \rightarrow \mathbb{C}$ by integrating $d F=\Phi$. Thanks to (iii), this map is an immersion and its image is an immersed exceptional flat surface with roof function given by $u=\operatorname{Re} U$. In 
Section 4, we will give some explicit examples of such constructions when $\partial \tilde{M}$ is equal to $\partial D \backslash\left\{\alpha_{1}, \ldots, \alpha_{n}\right\}$, where $\alpha_{1}, \ldots, \alpha_{n}$ is a finite collection of points on $\partial D=S^{1}$.

Example 3.1. Here is a (rather pathological) illustration of this Weierstrass-type formula. Consider $M=\mathbb{C}^{+}$, the function $U(z)=z$ and

$$
F(z)=\int_{0}^{z} e^{-\sinh \zeta} d \zeta
$$

Note that $\partial_{z} F$ is $2 i \pi$-periodic, and this implies that $F(z+2 i \pi)=F(z)+C$, where the constant $C$ is given explicitly by

$$
C:=i \int_{0}^{2 \pi} e^{-i \sin s} d s
$$

Moreover, for $x>0$,

$$
F(x+i y)=F(i y)+\int_{0}^{x} e^{-\sinh (s+i y)} d s
$$

converges to $+\infty$ as $x \rightarrow+\infty$ if $y=0$, but this quantity is bounded if $|y-\pi|<\pi / 2$, and even admits a finite limit as $x \rightarrow+\infty$.

Hence, in addition to the regular boundary $F(i \mathbb{R})$, which is a smooth periodic curve, the image of $F$ has a singular boundary: the set of limits of $F(x+i y)$ as $u$ tends to $+\infty$, for the values of $y$ for which this limit exists. The roof function tends to infinity along this singular boundary.

\section{Examples of exceptional flat surfaces}

Thanks to the Weierstrass-type representation in the previous section, we can give many nontrivial examples of exceptional flat surfaces. We keep the notation from that section.

The construction makes use of an integer $n \in \mathbb{N} \backslash\{0\}$ and the Riemann surface $D=\{z \in \mathbb{C}:|z|<1\}$. On $D$, we define the holomorphic functions

$$
h(z)=z^{n-1} \quad \text { and } \quad U(z):=\frac{1+z^{n}}{1-z^{n}} .
$$

The 1 -form $\Phi$ is given by

$$
\Phi(z):=\frac{2 n}{\left(1-z^{n}\right)^{2}} d z
$$

Both $U$ and $\Phi$ have singularities at the $n$-th roots of unity. The function $F$ is then obtained by integrating $\Phi$, and the roof function $u$ is defined by $u=\operatorname{Re} U$. 
(i) When $n=1$, we can take

$$
F(z)=\frac{1+z}{1-z}
$$

In this case, we simply have $F(D)=\mathbb{C}^{+}$, and we recover the fact that the half-plane is an exceptional domain. This is the counterpart of the plane in the framework of minimal surfaces.

(ii) When $n=2$, we can take

$$
F(z)=\frac{2 z}{1-z^{2}}+\log \frac{z+1}{z-1} .
$$

In this case, the exceptional flat surface found can be isometrically embedded in $\mathbb{C}$, and hence $F(D)$ is an exceptional domain. In fact, $F(D)$ corresponds (up to some similarity) to the domain $\Omega$, which was defined in Proposition 2.1. This exceptional domain is the counterpart of the catenoid.

(iii) Finally when $n \geq 3$, the exceptional flat surfaces we find cannot be isometrically embedded in $\mathbb{C}$ anymore. They are counterparts of the minimal $n$-noids described in [Jorge and Meeks 1983].

Let us analyze this example further. The function $U$ can be written as

$$
U(z)=-\frac{1}{n} \sum_{k=1}^{n} \frac{z+\alpha^{k}}{z-\alpha^{k}},
$$

where $\alpha:=e^{i 2 \pi / n}$. In particular, $\operatorname{Re} U$ is nothing but a multiple of the sum of the Poisson kernel on the unit disc with poles at $1, \alpha, \ldots, \alpha^{n-1}$. Next,

$$
d U=z^{n-1} \frac{2 n}{\left(1-z^{n}\right)^{2}} d z,
$$

so the function $h$ is cooked up to counterbalance the zero of $d U$ and ensure that $\Phi$ does not vanish in the unit disk, while keeping the condition $|d U|^{2}=|\Phi|^{2}$ on $\partial D$.

To generalize the example, consider $n$ distinct points $\alpha_{1}, \ldots, \alpha_{n} \in S^{1} \subset \mathbb{C}$ and $a_{1}, \ldots, a_{n}>0$. We define

$$
U(z):=-\sum_{k=1}^{n} a_{k} \frac{z+\alpha_{k}}{z-\alpha_{k}} .
$$

It is easy to check that $\operatorname{Re} U$ is positive (since each function $z \mapsto-\frac{z+\alpha_{k}}{z-\alpha_{k}}$ maps $D$ to $\mathbb{C}^{+}$) and vanishes on $\partial D \backslash\left\{\alpha_{1}, \ldots, \alpha_{n}\right\}$. We have

$$
\prod_{k=1}^{n}\left(z-\alpha_{k}\right)^{2} d U=P(z) d z,
$$


where $P$ is a polynomial that depends on the choice of points $\alpha_{1}, \ldots, \alpha_{n}$ and weights $a_{1}, \ldots, a_{n}$. Assume that $P$ does not vanish on $\partial D$ and denote by $z_{1}, \ldots, z_{l}$ the roots of $P$ in the unit disc, counted with multiplicity. We simply define

$$
h(z):=\prod_{j=1}^{l} \frac{z-z_{j}}{z \bar{z}_{j}-1}
$$

and the 1 -form $\Phi$ by $\Phi:=(1 / h) d U$. Integration of $\Phi$ yields a $2 n$-dimensional family of exceptional flat surfaces immersed in $\mathbb{C}$.

\section{A global Weierstrass-type representation}

In this section, we show that exceptional flat surfaces whose immersion in $\mathbb{C}$ have finitely many regular ends and are locally finite coverings of $\mathbb{C}$ are precisely the examples in the previous section. We use the notations introduced in Section 3, and we set

$$
\widehat{M}:=M \cup \partial M .
$$

We further assume that $M$ is simply connected and that $\partial M \neq \varnothing$. In particular, $M$ has the conformal type of the unit disk $D$, and without loss of generality, we can assume that $M$ is indeed equal to $D$ and consider $\bar{D}$ as a natural compactification of $M$. We denote by $F$ an orientation preserving, holomorphic, isometric immersion $F:(\widehat{M}, g) \rightarrow\left(\mathbb{C}, g_{\mathbb{C}}\right)$. Recall that $\|d F\|_{g}=1$ on $\partial M$. Some natural hypotheses are needed:

(H-1) $M$ has finitely many ends. This means that

$$
\partial M=\partial D \backslash \bigcup_{j=1}^{n} E_{j}=\bigcup_{j=1}^{n} I_{j},
$$

where each $E_{j} \subset S^{1}$ is a closed arc and $I_{j} \subset S^{1}$ is an open arc.

$(\mathrm{H}-2) F$ is proper. This means that $F(w)$ tends to infinity as $w$ tends to $\bigcup_{j=1}^{n} E_{j}$.

(H-3) Each end of $M$ is regular. This means the image of $I_{j}:=\left(\theta_{j}^{-}, \theta_{j}^{+}\right)$by $F$ is a curve $\Gamma_{j}$ asymptotically parallel to fixed directions at infinity. In other words, there exist two unit vectors $\tau_{j}^{-}$and $\tau_{j}^{+} \in S^{1} \subset \mathbb{C}$ such that

$$
\lim _{\theta \in I_{j}, \theta \rightarrow \theta_{j}^{ \pm}} \frac{F\left(e^{i \theta}\right)}{\left|F\left(e^{i \theta}\right)\right|}=\tau_{j}^{ \pm} .
$$

This is the case, for example, if we assume each $\Gamma_{j}$ has finite total curvature.

(H-4) The mapping $F$ is a locally finite covering. This means there exists $d \in \mathbb{N}^{*}$ such that, for any $z \in \mathbb{C}$, the cardinal of $\{\zeta \in M: F(\zeta)=z\}$ is at most $d$.

We now state the main result of this section. 
Theorem 5.1. Assume that $M$ is a simply connected exceptional flat surface and let $F: M \rightarrow \mathbb{C}$ be an isometric immersion. Further assume that $(\mathrm{H}-1)-(\mathrm{H}-4)$ holds and identify $M$ with $D$. Then there exist $\mu \in \mathbb{R}, n$ distinct points $\alpha_{1}, \ldots, \alpha_{n} \in S^{1}$ and $n$ constants $a_{1}, \ldots, a_{n}>0$ such that

$$
d F=e^{i \mu} \prod_{k=1}^{m} \frac{\bar{z}_{k} z-1}{z-z_{k}} d U
$$

where $z_{1}, \ldots, z_{m} \in \bar{D}$ denote the zeros of $d U$, counted with multiplicity, and where

$$
U(z):=-\sum_{j=1}^{n} a_{j} \frac{z+\alpha_{j}}{z-\alpha_{j}} \quad \text { in } \bar{D} .
$$

The proof is divided into a few lemmas and propositions. We start by analyzing the ends $E_{j}$ and show that they reduce to isolated points $\alpha_{1}, \ldots, \alpha_{n}$. Next we analyze the behavior of $F$ near the points $\alpha_{j}$ and show that $F$ does not have any essential singularity there. Then we proceed with the analysis of the function $U$ and show that it has the expected form. The proof is completed with the study of the function $h$.

As promised, we first analyze the sets $E_{j}$ :

Lemma 5.2. Under the assumptions of Theorem 5.1, there exists a finite number of points $\alpha_{1}, \ldots, \alpha_{n} \in \partial D=S^{1}$ such that $\widehat{M}=\bar{D} \backslash\left\{\alpha_{1}, \ldots, \alpha_{n}\right\}$.

Proof. We need to show that each interval $E_{j}$ is reduced to a point. This essentially follows from the fact that the capacity of $E_{j}$ vanishes.

Suppose, for a contradiction, that $E_{j}$ is an arc of positive arc length for some $j$, and take some $l \in(0, \pi / 2)$ and an arc $E \subset E_{j}$ of length $l$. Our problem being invariant under the action of fractional linear transformations of the unit disk, we can assume without loss of generality that $E$ is the image of $[-l / 2, l / 2]$ under $s \mapsto e^{i s}$. Reducing $l$ if necessary, we can also assume that the opposite arc $-E$, the image of $[-l / 2, l / 2]$ under $s \mapsto-e^{i s}$, is contained in $S^{1} \backslash \bigcup_{j=1}^{n} E_{j}$.

Recall that for any smooth function defined on $(a, b)$ which satisfies $f(b)=1$ and $f(a)=0$, we have

$$
1=f(b)-f(0)=\int_{a}^{b} f^{\prime}(s) d s \leq\left(\int_{a}^{b}\left(f^{\prime}\right)^{2}(s) d s\right)^{1 / 2} \sqrt{b-a} .
$$

If in addition, $b-a \leq 2$, we conclude that

$$
\int_{a}^{b}\left(f^{\prime}\right)^{2}(s) d s \geq \frac{1}{2}
$$


Now assume that we are given a smooth function $f: \bar{D} \rightarrow \mathbb{R}$ such that $f=1$ on $E$ and $f=0$ on $-E$. Using the previous inequality, we can write

$$
\int_{D}\|\nabla f\|_{g \mathbb{C}}^{2} d x d y \geq \int_{D \cap\{|x|<\sin (l / 2)\}}\left|\partial_{y} f\right|^{2} d x d y \geq \int_{|x| \leq \sin (l / 2)} \frac{1}{2} d x=\sin \frac{l}{2} .
$$

Given $R>r>0$, define $\chi: \mathbb{C} \rightarrow \mathbb{R}$ by

$$
\chi(z)=\left\{\begin{array}{cl}
0 & \text { if }|z| \leq r, \\
\frac{\log (|z| / r)}{\log (|z| / R)} & \text { if } r \leq|z| \leq R, \\
1 & \text { if } R \leq|z|,
\end{array}\right.
$$

and we define $f: D \rightarrow \mathbb{R}$ by $f:=\chi \circ F$. Since $F$ is conformal, we can write

$$
\int_{D}\|\nabla f\|_{g_{\mathbb{C}}}^{2} d x d y=\int_{D}\|\nabla f\|_{g}^{2} d \operatorname{vol}_{g} .
$$

Now, using (H-4), we conclude that

$$
\int_{D}\|\nabla f\|_{g}^{2} d \operatorname{vol}_{g} \leq d \int_{\mathbb{C}}\|\nabla \chi\|_{g_{\mathbb{C}}}^{2} d x d y=d \frac{2 \pi}{\log (R / r)} .
$$

Fixing $r>0$ large enough, we can ensure that $f$ is identically equal to 0 on $-E$. Using (H-2), we see that $f$ is identically equal to 1 on each $E_{j}$, and in particular on $E$. Therefore $f$ can be used in (5-1), which together with (5-2) yields

$$
2 \pi d \geq \sin \frac{l}{2} \log \frac{R}{r}
$$

independently of $R>r$. Letting $R$ tend to infinity, we get a contradiction, and the proof is complete.

Therefore, we now know that $E_{j}:=\left\{\alpha_{j}\right\}$. Without loss of generality, we can assume that $\alpha_{1}, \ldots, \alpha_{n}$ are arranged counterclockwise along $S^{1}$. We agree that $\alpha_{0}:=\alpha_{n}$ and $\alpha_{n+1}:=\alpha_{1}$, and that for each $j=1, \ldots, n$, the arc $I_{j}$ is positively oriented and joins $\alpha_{j}$ to $\alpha_{j+1}$. We now analyze the singularities of $F$ close to $\alpha_{j}$.

Given $j=1, \ldots, n$, we denote by $S\left(\alpha_{j}, r\right)$ the circle of radius $r>0$ centered at $\alpha_{j}$. We define

$$
\gamma_{j}:=\bar{D} \cap S\left(\alpha_{j}, r\right)
$$

which we assume to be oriented clockwise. The angle $\theta_{j} \in \mathbb{R}$ at $\alpha_{j}$ is defined by

$$
\theta_{j}:=-\lim _{r \rightarrow 0} \int_{\gamma_{k}} F^{*} d \theta
$$

where $d \theta:=\operatorname{Im} d z / z$. Thanks to (H-3), $\theta_{j}$ is well defined, and we have

$$
\tau_{j}^{-}=e^{i \theta_{j}} \tau_{j-1}^{+} \text {. }
$$


Lemma 5.3. Under the assumption of Theorem 5.1, the function

$$
H_{j}(z):=\left(z-\alpha_{j}\right)^{\theta_{j} / \pi} F(z)
$$

is holomorphic in a neighborhood of $\alpha_{j}$ in $\bar{D} \backslash\left\{\alpha_{j}\right\}$, and $H_{j}\left(\alpha_{j}\right) \neq 0$.

Proof. Without loss of generality, we can assume that $\alpha_{j}=1$. By right composing $F$ with the conformal transformation $z \mapsto(1-z) /(1+z)$, we can replace $D$ by $\mathbb{C}^{+}$. Now we define

$$
G(z):=F(z)^{-\pi / \theta_{j}} .
$$

Observe that $G(0)=0$ by $(\mathrm{H}-2)$. Moreover, $(\mathrm{H}-3)$ and the definition of $\theta_{j}$ imply that the image by $G$ of a neighborhood of 0 in $i \mathbb{R}$ is a $\mathscr{C}^{1}$-curve, and hence analytic. In particular, there exists some conformal transformation $T$ such that, for some $r>0$, the image by $T \circ G$ of $i(-r, r)$ is a straight line segment in $i \mathbb{R}$. Then it is possible to extend $T \circ G$ to a function $\tilde{G}$ defined on a neighborhood of 0 in $\mathbb{C}$ by setting

$$
\tilde{G}(z)= \begin{cases}T(G(z)) & \text { if } \operatorname{Im} z \geq 0 \\ -\overline{T(G(-\bar{z}))} & \text { if } \operatorname{Im} z \leq 0 .\end{cases}
$$

The resulting $\tilde{G}$ is bounded in a neighborhood of 0 in $\mathbb{C}$ and holomorphic away from 0. It is well known that the singularity is then removable and hence it is holomorphic. Therefore $\tilde{G}$ is actually holomorphic in a neighborhood of 0 . In particular, we can write

$$
G(z)=z^{k} H(z)
$$

near 0 , where $H$ is a holomorphic function that does not vanish at 0 . Going back to the definition of $G$, this implies that

$$
F(z)=\left(z-\alpha_{j}\right)^{-k \theta_{j} / \pi} H_{j}(z)
$$

where $H_{j}$ is holomorphic in a neighborhood of $\alpha_{j}$ and does not vanish at $\alpha_{j}$. But the definition of $\theta_{j}$ readily implies that $k=1$. This completes the proof.

As a corollary, we conclude that

$$
H(z):=F(z) \prod_{j=1}^{n}\left(z-\alpha_{j}\right)^{\theta_{j} / \pi}
$$

is a bounded holomorphic function in $\bar{D}$. Moreover, since $F$ tends to infinity as $z$ approaches $\alpha_{j}$, this implies that $\theta_{j}>0$.

We now make use of the fact that $M$ is an exceptional domain, and hence there is a roof function $u: \widehat{M} \rightarrow[0,+\infty)$. We can define the holomorphic function $U:=u+i v$, where $v: \widehat{M} \rightarrow \mathbb{R}$ is the (real-valued) harmonic conjugate of $u$. The purpose of the next result is to show that $U$ is precisely given by (4-1). 
Lemma 5.4. Under the assumptions of Theorem 5.1, there exist $n$ constants $a_{1}$, $\ldots, a_{n}>0$ such that

$$
U(z)=-\sum_{j=1}^{n} a_{j} \frac{z+\alpha_{j}}{z-\alpha_{j}}
$$

Proof. First, it is possible to extend the function $U$ to all $\mathbb{C} \backslash\left\{\alpha_{1}, \ldots, \alpha_{n}\right\}$ by defining $V$ to be equal to $U$ in $\bar{D} \backslash\left\{\alpha_{1}, \ldots, \alpha_{n}\right\}$, and

$$
V(z):=-\overline{U(1 / \bar{z})}
$$

when $z \in \mathbb{C} \backslash \bar{D}$. The key observation is that, since $\operatorname{Re} U=0$ on $\partial D \backslash\left\{\alpha_{1}, \ldots, \alpha_{n}\right\}$, the function $V$ is continuous and in fact holomorphic on $\mathbb{C} \backslash\left\{\alpha_{1}, \ldots, \alpha_{n}\right\}$. Moreover, $V$ converges to $V(\infty):=-\overline{U(0)}$ at infinity.

We proceed with the proof that the function $V$ has no essential singularity at any $\alpha_{j}$; it will follow from Picard's theorem. By definition, $\operatorname{Re} V$ vanishes on $I_{j}$ and is positive in $D$. Therefore the outward normal derivative of $\operatorname{Re} V$ on $I_{j}$ is negative. This implies that the tangential derivative of $\operatorname{Im} V$ on $I_{j}$ does not vanish and hence that $\operatorname{Im} V$ is strictly monotone on each $I_{j}$. This shows that there exists some neighborhood $\mathscr{V}$ of $\alpha_{j}$ in $\mathbb{C}$ such that any element of $i \mathbb{R}$ is achieved by $V$ at most twice in $\mathscr{V}$ (that is, at most once on $I_{j}$ and at most once on $I_{j-1}$ and certainly not in $\mathscr{V} \backslash \partial D$, since $V$ takes values in $\mathbb{C} \backslash i \mathbb{R}$ away from $\partial D$ ). Picard's big theorem [Conway 1978] then implies that $\alpha_{j}$ is not an essential singularity of $V$. Hence $\alpha_{j}$ is either a removable singularity of $V$ or a pole.

Since $\|\nabla u\|_{g} \equiv 1$ on $\partial M$, this forces $\left|\partial_{z} U\right|=\left|\partial_{z} F\right|$ on $\partial M$, and since $\left|\partial_{z} F\right|$ tends to $+\infty$ at $\alpha_{j}$, so does $\left|\partial_{z} U\right|$. Hence all $\alpha_{j}$ are poles of $V$.

We are now interested in the zeros of $V$. Since $\operatorname{Re} V$ takes positive values in $D$ and negative values in $(\mathbb{C} \cup\{\infty\}) \backslash \bar{D}$, we already know that the only possible zeros of $V$ are on $\partial D$. We have already seen that, along $I_{j}$, the function $V$ equals $i v$, where $v$ is strictly monotone. Further, since $\alpha_{j-1}$ and $\alpha_{j}$ are poles of this function, $|V|$ must converge to $+\infty$ as we approach either $\alpha_{j-1}$ or $\alpha_{j}$. Because of the continuity of $v$ along each $I_{j}$ it follows that $v$ vanishes exactly at one point $\beta_{j}$ on each $I_{j}$. Moreover, this zero is simple: if it had order $k>1$, the zero set of $\operatorname{Re} V$ near $\beta_{j}$ would contain $k$ curves intersecting at $\beta_{j}$, and this would force $\operatorname{Re} V=\operatorname{Re} U$ to vanish in $D$, in contradiction with our hypothesis.

Finally, we prove that $V$ has only simple poles. We know that $V$ extends meromorphically to a map on $\mathbb{C} P^{1}=\mathbb{C} \cup\{\infty\}$ with neither a pole nor a zero at infinity. Furthermore, $V$ has exactly $n$ simple zeros and $n$ poles; hence these poles must be simple. To summarize, $V$ can be written as a linear combination of the constant function and functions of the form $z \mapsto 1 /\left(z-\alpha_{j}\right)$. Without loss of generality, this 
amounts to saying that $V$ can be written as

$$
V(z)=a-\sum_{j=1}^{n} a_{j} \frac{z+\alpha_{j}}{z-\alpha_{j}},
$$

where $a$ and the $a_{j}$ are complex numbers. Using the fact that, by construction, $V(1 / \bar{z})=-\overline{V(z)}$, we conclude that $a \in i \mathbb{R}$ and also that $a_{j} \in \mathbb{R}$. Moreover, since $\operatorname{Re} U$ is positive, this implies that the $a_{j}$ are positive real numbers. This completes the proof, since $U$ is defined up to the addition of some element of $i \mathbb{R}$.

We are now in a position to complete our analysis of the function $F$. Since $F$ is an immersion, $d F \neq 0$ on $\widehat{M}$. Hence there exists a unique holomorphic function $h$ on $\widehat{M}$ such that

$$
\partial_{z} U=h \partial_{z} F
$$

on $\widehat{M}$. Moreover, since $\|\nabla u\|_{g} \equiv 1$ on $\partial M$, this implies that $|h| \equiv 1$ on $\partial M$. We now analyze the function $h$, which will complete the proof of Theorem 5.1.

Lemma 5.5. Under the assumptions of Theorem 5.1, there exists a constant $e^{i \mu} \in \mathbb{R}$ such that the function $h$ defined by (5-4) has the form

$$
h(z)=e^{-i \mu} \prod_{k=1}^{m} \frac{z-z_{k}}{\bar{z}_{k} z-1},
$$

where $z_{1}, \ldots z_{m}$ are the zeros of $\partial_{z} U$ in $D$ counted with multiplicity.

Proof. The function $h$ is holomorphic in $D$ and satisfies $|h|=1$ on $\partial D \backslash\left\{\alpha_{1}, \ldots, \alpha_{n}\right\}$. We can extend $h$ to a holomorphic function $H$, defined on $(\mathbb{C} \cup\{\infty\}) \backslash\left\{\alpha_{1}, \ldots, \alpha_{n}\right\}$ by setting $H(z):=h(z)$ for all $z \in \bar{D} \backslash\left\{\alpha_{1}, \ldots, \alpha_{n}\right\}$ and

$$
\overline{H(z)}:=\frac{1}{h(1 / \bar{z})}
$$

for all $z \in \mathbb{C} \backslash \bar{D}$. Clearly $H$ is locally bounded in $\bar{D} \backslash\left\{\alpha_{1}, \ldots, \alpha_{n}\right\}$, and its only singularities in $(\mathbb{C} \cup\{\infty\}) \backslash \bar{D}$ are poles that are the images by $z \mapsto 1 / \bar{z}$ of the zeros of $h$; hence $H$ is meromorphic outside $\left\{\alpha_{1}, \ldots, \alpha_{n}\right\}$. But Lemma 5.3 and (5-3) imply that, near $\alpha_{j},|H|$ is bounded by a constant times $\left|z-\alpha_{j}\right|^{-k_{j}}$ for some $k_{j}>0$. Therefore $\alpha_{j}$ is not an essential singularity of $H$, and hence $H$ is meromorphic in $\mathbb{C} \cup\{\infty\}$.

Observe that $|H(z)|=1$ on $\partial D \backslash\left\{\alpha_{1}, \ldots, \alpha_{n}\right\}$, and this implies that the points $\alpha_{j}$ are not poles of $H$. Therefore, the singularities $\alpha_{j}$ of $H$ are removable. Also, we have

$$
\Delta|H|^{2}=4 \partial_{z} \partial_{\bar{z}}|H|^{2}=4\left|\partial_{z} H\right|^{2} \geq 0,
$$

and since $|H|=1$ on $\partial D$, the maximum principle implies that $|H| \leq 1$ in $D$. 
Since $H$ is bounded in $\bar{D}$, it does not have poles in this set. This also implies that $H$ has no zeroes in $(\mathbb{C} \cup\{\infty\}) \backslash D$, because otherwise $H$ would have poles in $\bar{D}$ by (5-5). Therefore, if $z_{1}, \ldots, z_{m} \in D$ denote the zeros of $H$ (counted with multiplicity), the poles of $H$ are given by $1 / \bar{z}_{1}, \ldots, 1 / \bar{z}_{m}$ (also counted with multiplicity). It is then a simple exercise to check that $H$ is of the form

$$
H(z)=C \prod_{k=1}^{m} \frac{z-z_{k}}{\bar{z}_{k} z-1}
$$

for some constant $C \in \mathbb{C}$. Finally, the condition that $|H(z)|=1$ on $\partial D$ forces $|C|=1$. This completes the proof.

\section{A Bernstein type result for two-dimensional exceptional domains}

We prove the following Bernstein type result for two-dimensional exceptional domains.

Proposition 6.1. Assume that $\Omega$ is a two-dimensional exceptional domain conformal to $\mathbb{C}^{+}$, and let $u$ be a roof function on $\Omega$. We further assume that $\partial_{x} u>0$ in $\Omega$. Then $\Omega$ is a half-plane.

Proof. Since we have assumed that $\Omega$ is conformal to $\mathbb{C}^{+}$, there exists a holomorphic map $\Psi: \mathbb{C}^{+} \mapsto \Omega$. We then define

$$
H:=\left(\partial_{z} u\right) \circ \Psi .
$$

The function $H$ is holomorphic in $\mathbb{C}^{+}$and does not vanish, since we have assumed that $\partial_{x} u \neq 0$. Moreover, $|H| \equiv 1$ on $\partial \mathbb{C}^{+}$. We can write $H=e^{i \Theta}$, where $\Theta$ is a holomorphic function defined in $\mathbb{C}^{+}$that is real valued on the imaginary axis. This means that $\operatorname{Im} \Theta=0$ when $\operatorname{Re} z=0$. Since we have assumed that $\partial_{x} u>0$, we also conclude that $\operatorname{Re} \Theta \in(-\pi / 2, \pi / 2)$.

We can extend $\Theta$ as a holomorphic function $\tilde{\Theta}$ in $\mathbb{C}$ as follows:

$$
\tilde{\Theta}(z):= \begin{cases}\Theta(z) & \text { if } \operatorname{Re} z \geq 0, \\ \overline{\Theta(-\bar{z})} & \text { if } \operatorname{Re} z<0 .\end{cases}
$$

It is easy to check that $\tilde{\Theta}$ is a holomorphic function: in fact, the real part of $\Theta$ is extended as an even function of $\operatorname{Re} z$, while the imaginary part of $\Theta$ is extended as an odd function of $\operatorname{Re} z$. That $\tilde{\Theta}$ is $\mathscr{C}^{1}$ is then a consequence of the fact that $\operatorname{Im} \Theta=0$ on the imaginary axis, while the holomorphicity of $\Theta$ follows from the fact that $\partial_{x} \operatorname{Re} \Theta=0$ on the imaginary axis of $\mathbb{C}$.

The real part of $\tilde{\Theta}$, being a bounded harmonic function, must be constant. Then $\tilde{\Theta}$, being holomorphic, must itself be constant. But this implies that the gradient of $u$ is constant, and hence the level sets of $u$ are straight lines. This implies that 
$u$ only depends on one variable, and hence it is an affine function. This completes the proof.

Corollary 6.2. There is no exceptional domain contained in a wedge

$$
\Omega \subset\{z \in \mathbb{C}: \operatorname{Re} z \geq \kappa|\operatorname{Im} z|\}
$$

for any $\kappa>0$.

Proof. The proof is by contradiction. If $\Omega$ were such an exceptional domain, there would exist on $\Omega$ a roof function $u$. One can apply the moving plane method [Serrin 1971; Gidas et al. 1979] to prove that $\partial_{x} u>0$ and hence that $\partial \Omega$ is a graph over the $y$-axis. Since $\Omega$ is contained in a half-plane, there is no bounded, positive, harmonic function on $\Omega$ having 0 boundary data on $\partial \Omega$; otherwise one could use an affine function as a barrier to obtain a contradiction. Certainly, $\Omega \cup \partial \Omega$ is conformal to $\bar{D} \backslash E$, where $D$ is the unit disc and $E$ is a closed arc included in $S^{1}$. Necessarily, $E$ is reduced to a point, since otherwise we can construct bounded, positive, harmonic functions on $E$ that have 0 boundary data on $S^{1} \backslash E$, and these would lift to bounded, positive, harmonic function on $\Omega$, with 0 boundary data, a contradiction. Therefore, we conclude that $\Omega$ is conformal to $\mathbb{C}^{+}$. The assumptions of Lemma 5.5 are fulfilled, and hence we conclude that $\Omega$ is a half-plane, which is a contradiction.

\section{Open problems}

We have no nontrivial example of an exceptional domain in higher dimensions $\mathbb{R}^{m}$ for $m \geq 3$, besides the ones described in Section 2. In dimension $m=2$, it is tempting to conjecture that (up to similarity) the only exceptional domains that can be embedded in $\mathbb{R}^{2}$ are half-spaces, the complement of a ball and the example discussed in Section 2.

\section{References}

[Conway 1978] J. B. Conway, Functions of one complex variable, I, Graduate Texts in Math. 11, Springer, New York, 1978. MR 80c:30003 Zbl 0277.30001

[Gidas et al. 1979] B. Gidas, W.-M. Ni, and L. Nirenberg, "Symmetry and related properties via the maximum principle”, Commun. Math. Phys. 68:3 (1979), 209-243. MR 80h:35043 Zbl 0425.35020

[Jorge and Meeks 1983] L. P. Jorge and W. H. Meeks, III, "The topology of complete minimal surfaces of finite total Gaussian curvature”, Topology 22:2 (1983), 203-221. MR 84d:53006 Zbl 0517. 53008

[Pacard and Sicbaldi 2009] F. Pacard and P. Sicbaldi, "Extremal domains for the first eigenvalue of the Laplace-Beltrami operator", Ann. Inst. Fourier (Grenoble) 59:2 (2009), 515-542. MR 2521426 Zbl 1166.53029

[Serrin 1971] J. Serrin, "A symmetry problem in potential theory”, Arch. Ration. Mech. Anal. 43:4 (1971), 304-318. MR 48 \#11545 Zbl 0222.31007 
[Soufi 2007] A. El Soufi and S. Ilias, "Domain deformations and eigenvalues of the Dirichlet Laplacian in a Riemannian manifold", Illinois J. Math. 51:2 (2007), 645-666. MR 2342681 Zbl 1124. 49035

[Ye 1991] R. Ye, "Foliation by constant mean curvature spheres", Pacific J. Math. 147:2 (1991), 381-396. MR 92f:53030 Zbl 0722.53022

Received January 4, 2010.

LAURENT HAUSWIRTH

laurent.hauswirth@univ-mlv.fr

UNIVERSITÉ PARIS-EST MARNE-LA-VALLÉE

Laboratoire D’ ANAlyse et Mathématiques ApPliquées

CitÉ DESCARTES

5 BouleVARd DESCARTES

CHAMPS-SUR-MARNE

77454 MARNE-LA-VALLÉE CEDEX 2

FRANCE

http://perso-math.univ-mlv.fr/users/hauswirth.laurent

FRÉDÉRIC HÉLEIN

helein@math.jussieu.fr

UNIVERSITÉ PARIS DIDEROT-PARIS 7

INSTITUT DE MATHÉMATIQUES DE JUSSIEU, UMR CNRS 7586

UFR DE MATHÉMATiQues, CASE 7012

BÂTIMENT CHEVALERET

75205 PARIS CEDEX 13

FRANCE

http://www.math.jussieu.fr/ helein/

FRANK PACARD

Institut Universitaire de France et Centre de Mathématiques Laurent Schwartz UMR CNRS 7640

ÉCOle POlytechniQue

91128 PALAISEAU

FRANCE

frank.pacard@math.polytechnique.fr

http://www.math.polytechnique.fr/ pacard 


\title{
PACIFIC JOURNAL OF MATHEMATICS
}

\author{
http://www.pjmath.org \\ Founded in 1951 by
}

E. F. Beckenbach (1906-1982) and F. Wolf (1904-1989)

\section{EDITORS}

V. S. Varadarajan (Managing Editor)

Department of Mathematics

University of California

Los Angeles, CA 90095-1555

pacific@math.ucla.edu

Vyjayanthi Chari

Department of Mathematics

University of California

Riverside, CA 92521-0135

chari@math.ucr.edu

\section{Robert Finn}

Department of Mathematics Stanford University

Stanford, CA 94305-2125

finn@math.stanford.edu

Kefeng Liu

Department of Mathematics

University of California

Los Angeles, CA 90095-1555

liu@math.ucla.edu
Darren Long

Department of Mathematics

University of California

Santa Barbara, CA 93106-3080

long@math.ucsb.edu

Jiang-Hua Lu

Department of Mathematics

The University of Hong Kong

Pokfulam Rd., Hong Kong jhlu@maths.hku.hk

Alexander Merkurjev

Department of Mathematics University of California

Los Angeles, CA 90095-1555 merkurev@math.ucla.edu
Sorin Popa

Department of Mathematics

University of California

Los Angeles, CA 90095-1555

popa@math.ucla.edu

Jie Qing

Department of Mathematics

University of California

Santa Cruz, CA 95064

qing@ cats.ucsc.edu

Jonathan Rogawski

Department of Mathematics

University of California

Los Angeles, CA 90095-1555

jonr@math.ucla.edu

\section{PRODUCTION}

pacific@math.berkeley.edu

Silvio Levy, Scientific Editor Matthew Cargo, Senior Production Editor

ACADEMIA SINICA, TAIPEI

CALIFORNIA INST. OF TECHNOLOGY

INST. DE MATEMÁTICA PURA E APLICADA

KEIO UNIVERSITY

MATH. SCIENCES RESEARCH INSTITUTE

NEW MEXICO STATE UNIV.

OREGON STATE UNIV.

\section{SUPPORTING INSTITUTIONS}

STANFORD UNIVERSITY
UNIV. OF BRITISH COLUMBIA
UNIV. OF CALIFORNIA, BERKELEY
UNIV. OF CALIFORNIA, DAVIS
UNIV. OF CALIFORNIA, LOS ANGELES
UNIV. OF CALIFORNIA, RIVERSIDE
UNIV. OF CALIFORNIA, SAN DIEGO
UNIV. OF CALIF., SANTA BARBARA

UNIV. OF CALIF., SANTA CRUZ

UNIV. OF MONTANA

UNIV. OF OREGON

UNIV. OF SOUTHERN CALIFORNIA

UNIV. OF UTAH

UNIV. OF WASHINGTON

WASHINGTON STATE UNIVERSITY

These supporting institutions contribute to the cost of publication of this Journal, but they are not owners or publishers and have no responsibility for its contents or policies.

See inside back cover or www.pjmath.org for submission instructions.

The subscription price for 2011 is US \$420/year for the electronic version, and \$485/year for print and electronic.

Subscriptions, requests for back issues from the last three years and changes of subscribers address should be sent to Pacific Journal of Mathematics, P.O. Box 4163, Berkeley, CA 94704-0163, U.S.A. Prior back issues are obtainable from Periodicals Service Company, 11 Main Street, Germantown, NY 12526-5635. The Pacific Journal of Mathematics is indexed by Mathematical Reviews, Zentralblatt MATH, PASCAL CNRS Index, Referativnyi Zhurnal, Current Mathematical Publications and the Science Citation Index.

The Pacific Journal of Mathematics (ISSN 0030-8730) at the University of California, c/o Department of Mathematics, 969 Evans Hall, Berkeley, CA 94720-3840, is published monthly except July and August. Periodical rate postage paid at Berkeley, CA 94704, and additional mailing offices. POSTMASTER: send address changes to Pacific Journal of Mathematics, P.O. Box 4163, Berkeley, CA 94704-0163.

PJM peer review and production are managed by EditFLOW ${ }^{\mathrm{TM}}$ from Mathematical Sciences Publishers.

PUBLISHED BY PACIFIC JOURNAL OF MATHEMATICS

at the University of California, Berkeley 94720-3840

A NON-PROFIT CORPORATION

Typeset in IATEX

Copyright $(2011$ by Pacific Journal of Mathematics 


\section{PACIFIC JOURNAL OF MATHEMATICS}

Volume $250 \quad$ No. $2 \quad$ April 2011

Realizing profinite reduced special groups

VinCENT Astier and Hugo Mariano

On fibered commensurability

DANNy CALEGARI, Hongbin SUN and Shicheng WANG

On an overdetermined elliptic problem

LAURENT HaUswirTh, FrÉdÉRIC HÉLEIN and FranK PACARD

Minimal sets of a recurrent discrete flow

HATTAB HAWETE

Trace-positive polynomials

IGOR KLEP

Remarks on the product of harmonic forms

LiviU ORnea and Mihaela Pilca

Steinberg representation of GSp(4): Bessel models and integral representation of $L$-functions

Ameya Pitale

An integral expression of the first nontrivial one-cocycle of the space of long knots in $\mathbb{R}^{3}$

KEIICHI SAKAI

Burghelea-Haller analytic torsion for twisted de Rham complexes

GUANGXIANG SU

$K(n)$-localization of the $K(n+1)$-local $E_{n+1}$-Adams spectral sequences

TAKESHI TORII

Thompson's group is distorted in the Thompson-Stein groups

Claire Wladis

Parabolic meromorphic functions 